\title{
A New Perspective on Earth's Radiation Zone
}

\author{
Delong Chi \\ Beijing Solar Terrestrial Space Weather Institute, Beijing, China \\ Email: chidelong2016@sina.com
}

How to cite this paper: Chi, D.L. (2021) A New Perspective on Earth's Radiation Zone. Journal of Applied Mathematics and Physics, 9, 2979-2986. https://doi.org/10.4236/jamp.2021.911191

Received: November 2, 2021

Accepted: November 27, 2021

Published: November 30, 2021

\begin{abstract}
The discovery of Earth's radiation zone divided into two belts is the first major discovery in Human space exploration. The inner belt dominated by energetic protons and the outer belt dominated by energetic electrons imply the inner belt with net positive charge and the outer belt with net negative charge. Earth's surface carries net negative charge. These exploration data suggest the inner and outer parts of a star may be composed of several discrete charged layers. We propose the mechanism of the star's multiple Debye spherical layers to adapt to the objective existence. The charged particles in the near-earth space follow the statistical distribution of multiple Debye spherical layers of Earth. The inner belt and outer belt is the main body of layer 8 and layer 9 respectively. The empty slot between the inner and outer belt comes from the action of the electric field between them. An impenetrable barrier to ultra-relativistic electrons originates from the action of the electric field of layer 7.
\end{abstract}

\section{Keywords}

Radiation Zone, Slot, Barrier

\section{Introduction}

The discovery of Earth's radiation zone divided into two belts is the first major discovery in Human space exploration. However, up to now, there is no perfect explanation for the cause of Earth's radiation zone being divided into two belts. In addition, the obvious empty slot between the inner and outer belt, an impenetrable barrier to ultra-relativistic electrons from the outer radiation belt to the inner radiation belt.

We have proposed a new mechanism of matter distribution. It can be used to explain the causes of these phenomena well.

Due to the release of gravitational potential energy and fusion energy, the center of a star is usually at a high temperature at which matter is ionized. Due 
to the electron diffusivity much higher than that of ions, Debye Sheath, i.e., electric double layer, the center-net positive charge region and the periphery-net negative charge region is formed. The electric field of the negative layer continues to act outward, resulting in the formation of the multiple Debye spherical layers of the star.

Inner parts and outer parts of Earth follow the statistical distribution of multiple Debye spherical layers of Earth. The inner belt and outer belt is the main body of layer 8 and layer 9 respectively. The empty slot between the inner belt and outer belt comes from the action of the electric field between them. An impenetrable barrier to ultra-relativistic electrons from the outer radiation belt to the inner radiation belt in the Van Allen radiation belts originates from the action of the electric field of layer 7.

\section{The Exploration Data and Analysis}

From 4 October 1957, Human beings have opened the era of space exploration, especially discovered Earth's radiation zone and Earth's radiation zone is divided into two belts, the inner belt dominated by energetic protons and the outer belt dominated by energetic electrons, the obvious empty slot between the inner belt and outer belt, an impenetrable barrier to ultra-relativistic electrons from the outer radiation belt to the inner radiation belt [1]

In areas of fair weather, the atmospheric electric field near Earth's surface typically is about 100 volts $(\mathrm{V}) \mathrm{m}^{-1}$ and is directed vertically downward to Earth [2].

The inner belt dominated by energetic protons and the outer belt dominated by energetic electrons, suggest the inner belt with net positive charge and the outer belt with net negative charge.

The atmospheric electric field is directed vertically downward to Earth suggests the atmospheric electric field: 1) only originates from crust with net negative charges 2 ) only originates from ionosphere with net positive charges 3 ) originates from electric double layer: crust with net negative charge and the ionosphere with net positive charge.

The above analyses of exploration data strongly suggest that the inner and outer parts of the Earth may be composed of several discrete charged layers. In this situation, we reveal its possibility and promote space exploration in the following argument and discussion.

\section{Debye Sheath-Electric Double Layer}

Since:

1) Due to the release of gravitational potential energy and nuclear fusion energy inside Earth, Earth's center is at a high temperature at which matter is ionized and emerges a large temperature difference between Earth's center and its periphery [3] [4] [5] [6].

2) Set $T_{\mathrm{e}} \approx T_{i}$ in Earth's center, the ratio of electron thermal rate $v_{\mathrm{e}}$ to ion 
thermal rate $v_{i}, \frac{v_{\mathrm{e}}}{v_{i}} \approx\left(\frac{m_{i}}{m_{e}}\right)^{\frac{1}{2}}$, then Ratio of electron diffusivity $D_{\mathrm{e}}$ to ion diffusivity $D_{i}, \frac{D_{\mathrm{e}}}{D_{i}} \approx \frac{v_{\mathrm{e}}}{v_{i}} \approx\left(\frac{m_{i}}{m_{e}}\right)^{\frac{1}{2}}>1$.

Then:

As a statistical result of the thermal motion of the charged particles in Earth's center and its periphery, Debye sheath, i.e., electric double layer, one net positively charged region $\left(q_{0}\right)$-positive layer in Earth's core and one net negatively charged region $\left(-q_{0}\right)$-negative layer in the periphery of Earth's core emerges [7]-[12]. As shown in Figure 1(a).

The positive layer is a group of particles dominated by the positive particles (such as the positive ions), the negative layer is a group of particles dominated by the negative particles (such as the electrons and the negative ions).

\section{Multiple Debye Spherical Layers of Earth}

In fact, when Debye sheath, electric double layer emerges in Earth's center and the periphery of Earth's center, the negative particles in the negative spherical

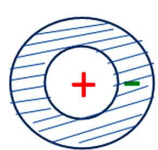

(a)

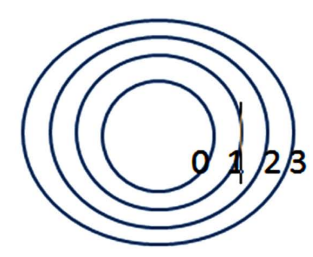

(c)

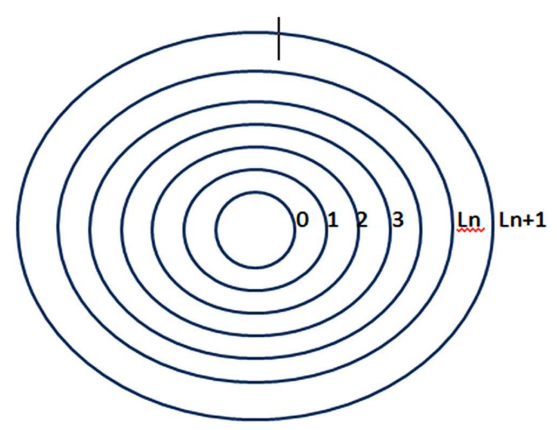

(b)

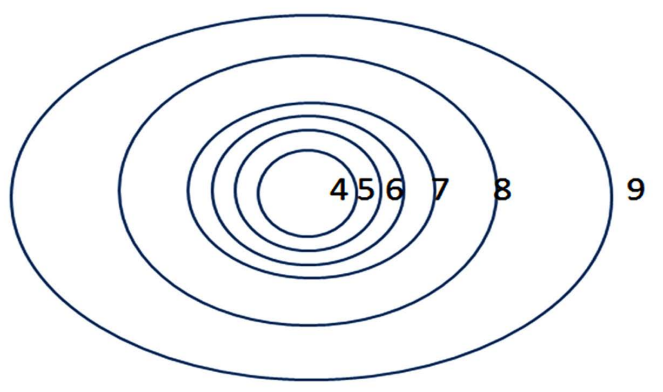

(d)

Figure 1. Debye sheath and Multiple Debye spherical layers of Earth. (a) Debye sheath, i.e., electric double layer: Earth's center (+)-layer 0; the periphery of Earth's center (-)-layer 1; (b) The corresponding equivalent charged thin spherical shells of Earth's multiple Debye spherical layers; (c) The corresponding equivalent charged thin spherical shells of Earth's multiple Debye spherical layers (inside Earth); (d) The corresponding equivalent charged thin spherical shells of Earth's multiple Debye spherical layers (outside Earth, $L \leq 10 \mathrm{R}_{\text {earth }}$ ). 
layer of Debye sheath attract inward the positive particles in the positive layer of the Debye sheath, meanwhile, the negative particles in the negative layer of the Debye sheath attract the positive and repel the negative particles in the periphery of the negative spherical layer. As a statistical motion result, another one positive spherical layer dominated by the positive particles emerges around the negative spherical layer. In the same way, another one negative spherical layer dominated by the negative particles also emerges around the positive spherical layer. In the same way, in turn One by one, net charged spherical layer $n+1$ emerges around the net charged spherical layer $n$. The adjacent layers (layer $n$, layer $n+1$ ) carry opposite sign charges, $\mathrm{n}$ is a natural number.

Due to Debye sheath, electric double layer, Earth's multiple Debye spherical layers generate. As shown in Figure 1(b).

The inner and outer matter of Earth follows the statistical distribution of Earth's multiple Debye spherical layers.

\section{Main Characteristics of Earth's Multiple Debye Spherical Layers}

1) The adjacent layers (layer $n$, layer $n+1$ ) carry opposite sign charges, an electric field forms between adjacent layers.

2) Due to the electric field between adjacent layers, a spherical capacitor forms between adjacent layers to store electrical energy. The gravitational potential energy and the fusion energy inside Earth can be transformed into the electric energy stored between adjacent layers of Earth's multiple Debye spherical layers.

3) The energy release between adjacent layers can drive the material movement in the corresponding space

4) The charged particles between adjacent layers will be expelled by the electric field between adjacent layers. The charged particles between adjacent layers, the negative particles among them will run to the positive layer and the positive particles will run to the negative layer. Finally, a slot will form between adjacent layers.

5) The thickness of each layer of Earth's multiple Debye spherical layers is positively related to the magnitude of $q_{0}$, inversely related to the space number density of charged particles. The space number density of layers in the multiple Debye spherical layers is inversely related to $q_{0}$ and positively related to the space number density of charged particles.

6) Except layer 0 and layer 1 , layer $n+1$ forms in the electric field of layer $n, n$ is a natural number. The $(+)$ layer is dominated by the positively charged particle, the (-) layer is dominated by the negatively charged particle.

7) Layer $n+1$ and layer $n$ carry opposite sign net charge, Layer $n+2 m$ and Layer $n$ carry same sign net charge, Layer $n+2 m+1$ and layer $n$ carry opposite sign net charge, $\mathrm{m}$ and $\mathrm{n}$ is a natural number.

8) The motion of the dominant particles of layer $n+2$ from layer $n+2$ to Layer $n+1$, or the motion of the dominant particles of layer $n+1$ from layer $n$ 
+1 to Layer $n+2$ is inhibited by the electric field of Layer $n, n$ is a natural number.

\section{The Matter Distribution and the Possible Corresponding Layer in Earth's Multiple Debye Spherical Layers}

At first, Earth's core is in (+) layer, the periphery of Earth's core is in (-) layer. Secondly, crust is in (-) layer, the inner radiation belt is in $(+)$ layer, the outer radiation belt is in (-) layer. Thirdly, there may be $2 n$ layers between Earth's core and crust. There may be $2 \mathrm{n}$ layers between crust and the inner radiation belt. $\mathrm{n}$ is a natural number.

The lower mantle may be in layer $1(-)$, the upper mantle may be is in layer 2 $(+)$, crust may be in layer $3(-)$ (Figure $1(c)$ ). Ionosphere D may be in layer 4 $(+)$, ionosphere E may be in layer $5(-)$, ionosphere F1 may be in layer $6(+)$, ionosphere F2 may be in layer $7(-)$. Although the shape of the radiation belts is not spherical, the main part of inner radiation belt may be in layer 8 or layer $2 \mathrm{n}$ (+) layer, the main part of outer radiation belt may be in layer 9 or layer $2 n+1$ (-) layer, as shown in Figure 1(d).

1) Energy release between adjacent layers of Earth's multiple Debye spherical layers can drive the material movement in the corresponding space. Such as mantle convection and crust motion, as well as atmosphere and ocean motion.

2) The disappearance of ionosphere $D$ and F1 at night does not mean that layer 4 and layer 6 also disappears. Since the space number density of charged particles decreases greatly near earth' surface at night, then although the thickness of some layers increases greatly near earth' surface at night, the penetration of electromagnetic waves surges, especially in layers 4,6 .

3) Whether during geomagnetic quiet period or during geomagnetic disturbance period, due to strongly coupled motion of upper atmosphere and ionosphere [13], no obvious slot between layers $4,5,6,7$, no obvious boundary of ionosphere D, E, F1, F2.

\section{Discussion}

1) The charged particles in the near earth space follow the statistical distribution of the multiple Debye spherical layers of Earth. At same time, the charged particles in the near earth space are greatly affected by the geomagnetic field. Geomagnetic field can greatly changes the multiple Debye spherical layers of Earth in the near earth space. The main part of inner radiation belt is in layer 8 -layer $2 \mathrm{n}(+)$ layer, the main part of outer radiation belt is in layer 9-layer $2 \mathrm{n}+1$ (-) layer. The inner belt and outer belt can be changed to be the main body of layer 8 and layer 9 respectively during geomagnetic quiet period, even the inner belt and outer belt can replace the corresponding layer respectively during geomagnetic quiet period, i.e., the inner belt and outer belt right is layer 8 and layer 9 respectively during geomagnetic quiet period (Figure 2).

2) In the three states, a) the main part of inner radiation belt is in layer 8-layer 
$2 \mathrm{n}(+)$ layer, the main part of outer radiation belt is in layer 9-layer $2 \mathrm{n}+1(-)$ layer, b) the inner belt and outer belt is the main body of layer 8-layer $2 \mathrm{n}(+)$ layer and t layer 9-layer $2 \mathrm{n}+1(-)$ layer respectively, c) the inner belt and outer belt right is layer 8-layer $2 \mathrm{n}(+)$ layer and layer 9-layer $2 \mathrm{n}+1(-)$ layer respectively, there is an electric field between inner and outer belt.

3) Due to the electric field between the inner and outer radiation belts, an slot of particle distribution between inner and outer radiation belts is formed during geomagnetic quiet period, i.e., an obviously empty slot between the inner and outer belts during geomagnetic quiet period.

4) The charged particles are free to move along the lines of magnetic field $\mathbf{B}$ [14]. The lines of geomagnetic field B pass through the adjacent layers of Earth's multiple Debye spherical layers. Since the adjacent layers carry opposite sign charges, then the current can emerge between the adjacent layers along the lines of geomagnetic field $\mathbf{B}$.

5) According to the configuration and flux of the magnetic field lines passing through adjacent layers, the current surface density between adjacent layers positively correlated the geomagnetic latitude.

6) Since the current between adjacent layers weakens the adjacent layer and draws the adjacent layer closer, i.e., the current between adjacent layers leads the layer thickness and the slot between adjacent layers to reduce or shrink, then the thickness of belt is inversely related to the geomagnetic latitude. The width of the empty slot between the inner and outer belt is also inversely related to the geomagnetic latitude, as shown in Figure 2.

7) a) During the period of strong geomagnetic activity, since the large amount of charged particles form solar wind injected into the near earth space, the space number density of charged particles increases greatly in the near earth space, the space number density of layers of the multiple Debye spherical layers of Earth also increases in the near earth space. This can lead (a) the disappearance of the slot between the inner and outer radiation belts, b) more radiation belts appears

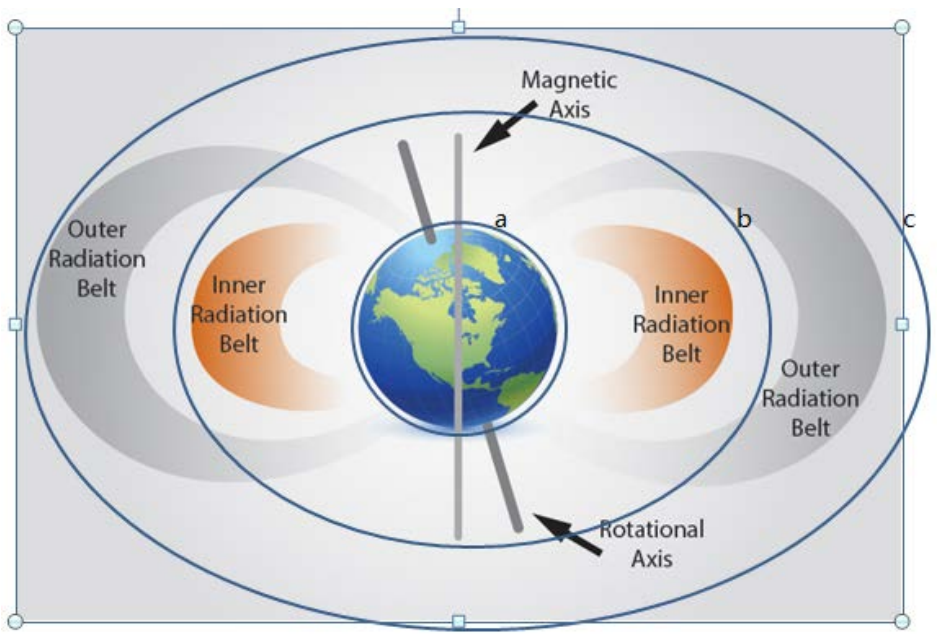

Figure 2. Earth's radiation zone in earth's multiple Debye spherical layers. 
in near earth space, i.e., the generation of the new layer. Since the high space number density of charged particles in near earth space is just transient state, then the existence of new radiation belt is also transient [15].

8) The electrons is negatively charged particles, dominating particles of layer 9 (-) layer, the motion of ultra-relativistic electrons from the outer radiation belt-layer $9(-)$ layer to the inner radiation belt-layer $8(+)$ layer is repelled by layer 7 (-) layer, i.e., is inhibited by the electric field of layer 7 (-) layer due to the action of Coulomb's force. This inhibition to ultra-relativistic electrons right is an impenetrable barrier from the outer radiation belt to the inner radiation belt in the Van Allen radiation belts.

Earth's radiation zone is in Earth's multiple Debye spherical layers. a, b, c may be the boundary of layers 8, 9, as shown in Figure 2.

\section{Conclusion}

Earth's radiation belts are a group of energetic particles, following the statistical distribution of Earth's multiple Debye spherical layers, strongly affected by the geomagnetic field. The exploration data on Earth's radiation belts can be well explained with the mechanism of Earth's multiple Debye spherical layers.

\section{Acknowledgements}

Special thanks to Professor Biping Gong, Professor Xiaotian Li, Professor Hefa Lv, My Assistant Jingsen Wu and all who supported me.

\section{Conflicts of Interest}

The authors declare no conflicts of interest regarding the publication of this paper.

\section{References}

[1] Baker, D.N. and Panasyuk, M.I. (2017) Discovering Earth's Radiation Belts. Physics Today, 70, 46. https://physicstoday.scitation.org/doi/10.1063/PT.3.3791 https://doi.org/10.1063/PT.3.3791

[2] https://www.feynmanlectures.caltech.edu/II_09.html

[3] https://ugc.berkeley.edu/background-content/earths-internal-heat

[4] Smylie, D.E. (2013) Earth Dynamics. 410. https://doi.org/10.1017/CBO9781139025409

[5] Stixrude, L. and Wasserman, E. (1997) Composition and Temperature of Earth's Inner core. Journal of Geophysical Research. https://doi.org/10.1029/97JB02125

[6] Tarbuck, E.J. and Lutgens, F.K. (2017) Earth Science. 15th Edition, 16-18.

[7] https://www.chemeurope.com/en/encyclopedia/Debye_sheath.html

[8] Plasmahtml-University of Texas at Austin. https://farside.ph.utexas.edu/teaching/plasma/lectures1/Plasmahtml.html

[9] Ishiguro, S., Kamimura, T. and Sato, T. (1985) Double Layer Formation Caused by Contact between Different Temperature Plasmas. Physics of Fluids, 28, 2100. https://doi.org/10.1063/1.865390 
[10] Langmui, I. (1923) Positive Ion Currents from the Positive Column of Mercury Arcs. Science, 58, 290-291. https://doi.org/10.1126/science.58.1502.290

[11] Hull, L.A. and Langmui, I. (1929) Control of an Arc Discharge by Means of a Grid. Proc Natl Acad Sci USA, 15, 218-225. https://doi.org/10.1073/pnas.15.3.218

[12] Hultqvist, B. (1971) On the Production of a Magnetic-Field-Aligned Electric Field by the Interaction between the Hot Magnetospheric Plasma and the Cold Ionosphere. Planetary and Space Science, 19, 749-759. https://doi.org/10.1016/0032-0633(71)90033-X

[13] Shinagawa, H., Jin, H., Miyoshi, Y., Fujiwara, H., Tanaka, T., Fujita, S., Terada, K. and Murata, K.T. (2011) Whole Atmosphere-Ionosphere Coupled Model (GAIA) for Space Weather Research, AGU Fall Meeting Abstracts.

[14] Chen, F.F. (2016) Introduction to Physics and Controlled Fusion. 3rd Edition, 359. https://doi.org/10.1007/978-3-319-22309-4

[15] https://www.space.com/20004-earth-radiation-belt-discovery.html 